\title{
BETATRON SIDEBANDS DUE TO ELECTRON CLOUDS UNDER COLLIDING BEAM CONDITIONS
}

\author{
J.W. Flanagan, K. Ohmi, H. Fukuma, Y. Funakoshi, S. Hiramatsu, H. Ikeda, M. Tobiyama, \\ S. Uehara, S. Uno, KEK, Tsukuba, Japan, and E. Perevedentsev, BINP, Novosibirsk, Russia
}

\begin{abstract}
Recently, we have observed vertical betatron sidebands in the transverse beam spectra of positron bunches at the KEKB LER which are associated with the presence of electron clouds.[1] When the LER is operating in single-beam mode (no colliding bunches in the HER), these sidebands are sharply peaked. When the bunches are in collision for physics running, the sidebands are still present but are found to be smeared out. The bunch-by-bunch specific luminosity is lower for bunches with sidebands than for those without sidebands. In this paper, the behavior of the sidebands in collision and the effects on luminosity are discussed.
\end{abstract}

\section{INTRODUCTION}

The KEKB accelerator is made up of two intersecting storage rings, the Low Energy Ring (LER) carrying 3.5 $\mathrm{GeV}$ positron bunches, and the High Energy Ring carrying $8 \mathrm{GeV}$ electron bunches. The LER has been subject to the build-up of electron clouds, which cause transverse beamsize blowup, and an accompanying loss of luminosity. The electron cloud build-up is partially suppressed by the use of solenoid magnets wound around the drift spaces of the LER beam pipe, and the luminosity does not appear to be limited by electron cloud-induced blow-up at the present LER beam current of $1.72 \mathrm{~A}$. The currently employed fill pattern is composed of bunches spaced an average of 3.5 buckets ( $\sim 7 \mathrm{~ns}$ ) apart. To achieve significantly higher beam currents, it will be necessary to reduce the bunch spacing further; however, one problem with doing this is that the specific luminosity has been found to be reduced for bunches at shorter bunch spacings. Electron-cloud build-up is suspected as the cause of this reduction in specific luminosity.

Recently, we have observed vertical betatron sideband peaks in the transverse beam spectra of LER bunches which appear to be a signature of head-tail instability caused by electron clouds.[1] These peaks appear to the right of the vertical betatron peak at and above the threshold current of electron cloud-induced vertical beam blow-up, and constitute a sensitive diagnostic for the presence of such cloudinduced instabilities. We have conducted some measurements on closely ( 2 bucket, $\sim 4 \mathrm{~ns}$ ) spaced bunches at varying bunch currents to investigate the relation between sideband peak height and specific luminosity, which we report on here. (Bunch-by-bunch beam sizes were also measured, as reported elsewhere in these proceedings.[2]) We also show spectra for bunches in and out of collision.

\section{OBSERVATIONS}

\section{Sidebands in and out of collision}

Vertical bunch position spectra were measured using 6 $\mathrm{mm}$ diameter BPMs, as described in [1]. All spectra were taken passively - there was no intentional excitation supplied.

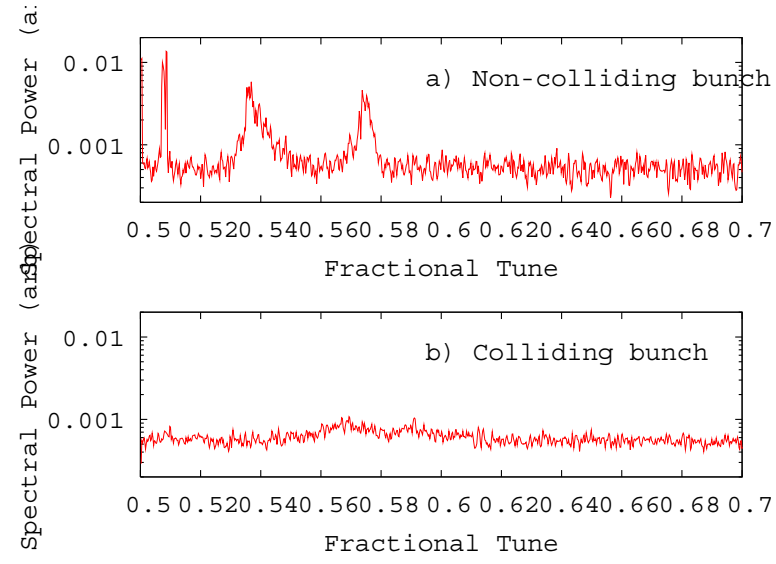

Figure 1: Bunch spectra for a) non-colliding, and b) colliding LER bunches with a 2-bucket (4 ns) separation from the preceeding bunch

Fig. 1 shows two sets of averaged bunch spectra. Fig. 1a shows the averaged spectra for bunches which have been placed $2 \mathrm{rf}$ buckets ( $4 \mathrm{~ns}$ ) behind regular bunches during physics operation. No corresponding bunch has been injected in the HER, so this is a non-colliding bunch. The LER bunch currents are $1.2 \mathrm{~mA}$. Two broad peaks are visible in the spectrum: the vertical betatron peak at the vertical tune of 0.536 , and the sideband peak at a tune of 0.575 . (In general, the betatron peak is not always visible, depending partly on the tuning of the feedback system at the time of measurement.) The peak at $\sim 0.0508$ is the horizontal tune, which also sometimes appears and sometimes does not.

The presence of the sideband peak shows that this combination of bunch current and spacing is above the electron cloud instability threshold, even though the beampipe solenoids are on. No such sidebands were observed in bunches with 3- or 4-bucket spacings in front of them.

Fig. 1b shows similarly-situated bunches, but in collision with corresponding bunches in the HER. The peaks are shifted to the right, and smeared out due to beam-beam tune spread. The peak heights are greatly reduced. 


\section{Sidebands and specific luminosity}

Next we measured the relationship between bunch current and specific luminosity for bunches with 2-bucket spaces between them and their preceeding bunches. (We call these bunches "2-bucket bunches" for brevity.) The current standard physics run pattern is made up of bunches alternating between 3-bucket spacing from their preceedings bunches ("3-bucket bunches") and 4-bucket spacing ("4-bucket bunches"), for an average bunch spacing of 3.5 buckets. On May 5, at one location in the fill pattern we inserted an extra bunch in the LER only, as a 2-bucket bunch, which was a non-colliding test bunch. Elsewhere in the fill pattern we inserted another 2-bucket bunch in both the LER and HER, to create a colliding test bunch. Both bunches were injected at the same current as the surrounding bunches (1.2 $\mathrm{mA}$ in the LER), and were then allowed to decay. The regular fill pattern bunches were kept topped up as part of the continuous injection mode of operation.

The luminosity of the colliding test bunch was measured using the bunch-by-bunch zero-degree luminosity monitor (ZDLM), which uses lead glass and a PMT to measure recoil electrons from radiative Bhabha events at the interaction point. Due to the smearing of the sideband peak under collision, we could not measure the sideband peak of the colliding test bunch directly, but monitored that of the noncolliding test bunch as it decayed in parallel.

The sideband peak height (in arbitrary units) as a function of test bunch current is plotted in Fig. 2. The peak height drops rapidly as the bunch current decays from 1.2 $\mathrm{mA}$ to just below $0.8 \mathrm{~mA}$, below which the sideband peak disappears (indicated by zero peak heights). The tune of the sideband peak, shown in Fig. 3, does not change.

Streak camera measurements, made at the same time,[2] indicate that the vertical beam size of the test bunches grow above $0.8 \mathrm{~mA}$, consistent with the electron-cloud instability threshold indicated by the sideband measurements.

The specific luminosity of the (2-bucket) colliding test bunch as a function of bunch current is shown in Fig. 4, along with those of a representative 3-bucket bunch and 4bucket bunch from the regular fill pattern. The trend line for the 2-bucket bunch is seen to be lower than that of the the 3and 4-bucket bunches at higher currents, but the trend lines join just below $0.8 \mathrm{~mA}$. This agreement with the sideband and beam size thresholds indicates that the loss of specific luminosity for the 2-bucket bunch relative to the 3- and 4bucket bunches is indeed due to electron cloud instability.

Another set of measurements were made on May 6, again with test bunches inserted into the regular physics fill pattern, but this time watching the beam spectra and luminosities of the bunches 2 buckets behind the test bunches, which we label as "observer bunches." The HER bunch corresponding to one of the LER observer bunches was removed, making that observer bunch the "non-colliding observer bunch." The other LER observer bunch was a colliding observer bunch. Again, the test bunches ( 2 buckets in front of the observer bunches) were allowed to decay,

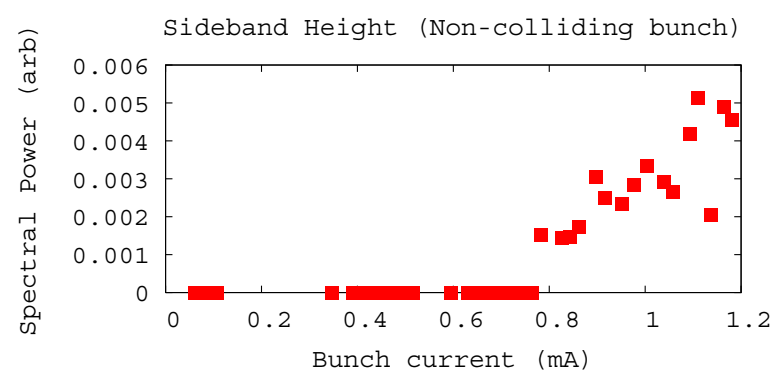

Figure 2: Sideband peak height of non-colliding test bunch as a function of test bunch current

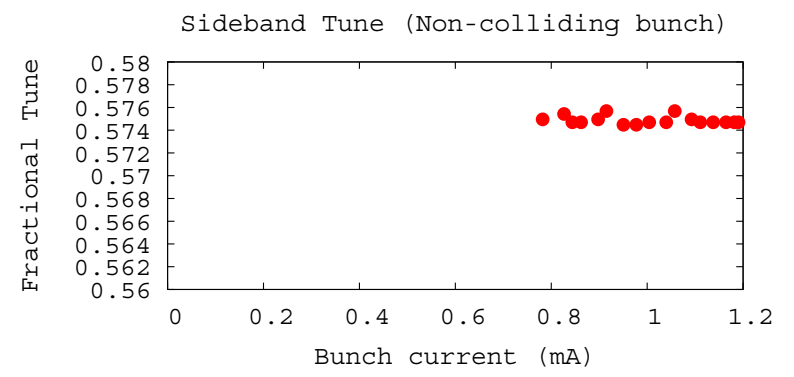

Figure 3: Sideband tune of non-colliding test bunch as a function of test bunch current

but the observer bunches were kept topped up along with the rest of the regular fill pattern. In other words, in this (May 6) measurement, the electron cloud density due to the preceeding bunch decreased while the current of the bunch under observation remained constant, whereas in the May 5 measurement, the electron cloud density was held constant while the current of the bunch under observation decayed.

Bunch spectra of the non-colliding observer bunch at various test bunch currents are shown in Figs. 5a-d. Each

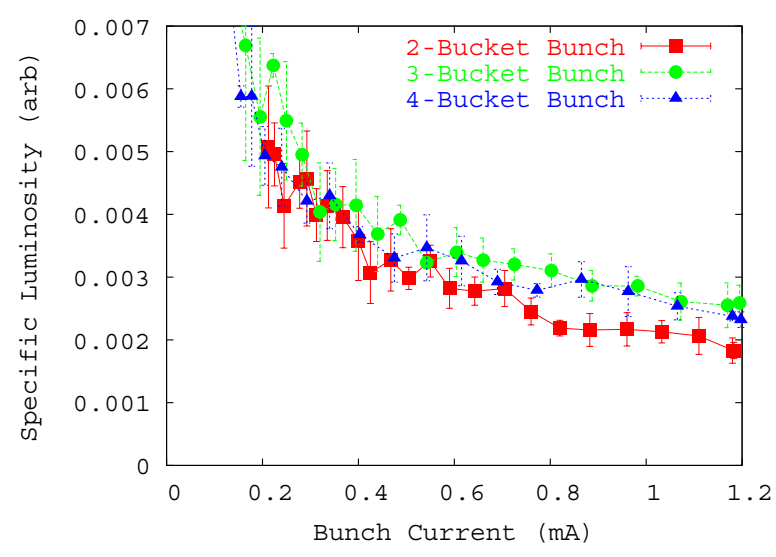

Figure 4: Specific luminosity as a function of bunch current for the 2-bucket colliding test bunch, as well as that for nearby 3-bucket and 4-bucket bunches. Each point is the average of 4 consecutive measurements; error bars are 1- $\sigma$ statistical error bars. 


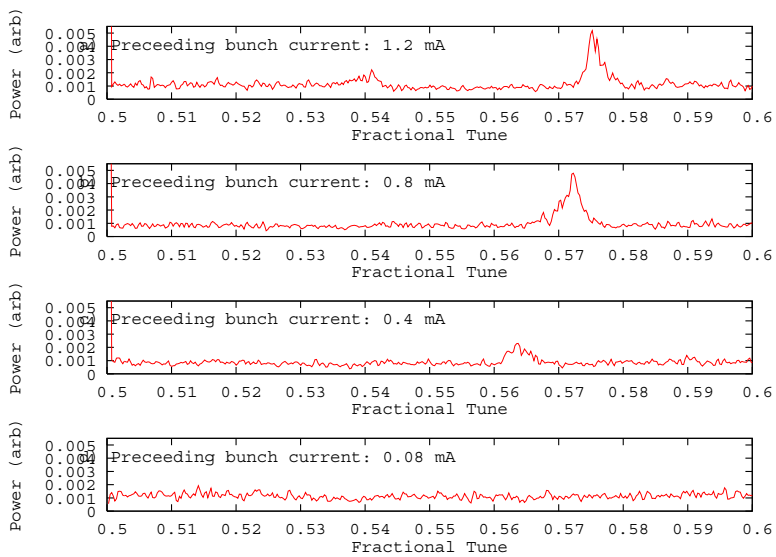

Figure 5: Bunch spectra of the non-colliding observer bunch. Figures a-d show the spectrum for the observer bunch at different bunch currents for the preceeding bunch (the test bunch).

plot is the average of 5 spectra taken for the same bunch. In Fig. 5a, at the highest bunch current, the sideband peak is visible at a tune of 0.575 . As the current decays, the sideband peak shifts to the left and decreases in height, disappearing entirely between the bunch currents of $0.4 \mathrm{~mA}$ and $0.08 \mathrm{~mA}$. (The vertical betatron peak at 0.54 is also visible at the highest current in this particular series.)

The sideband height and tune of the non-colliding $o b$ server bunch are plotted as functions of the preceeding test bunch in Figs. 6 and 7, respectively. The peak height decays until the test bunch current is just below $0.4 \mathrm{~mA}$. At that point, the test bunch suffered a lifetime loss, and there is a gap between just below $0.4 \mathrm{~mA}$ and $\sim 0.1 \mathrm{~mA}$. Below the gap, no sideband peak is seen. The sideband tune, in contrast to that in the May 5 measurement, gradually shifts down as the test bunch current decays.

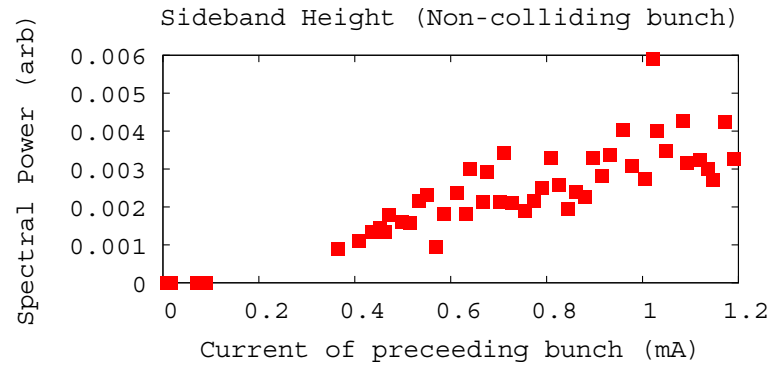

Figure 6: Sideband peak height of non-colliding observer bunch for different currents of preceeding bunch

The specific luminosity of the (2-bucket) colliding observer bunch and those of some representative 3- and 4bucket bunches are all plotted as functions of the current of the test bunch (which is 2 buckets in front of the observer bunch). The 3- and 4-bucket bunches show, of course, no dependence on the current of the test bunch, while the specific luminosity of the colliding observer bunch is lower
Sideband Tune (Non-colliding bunch)

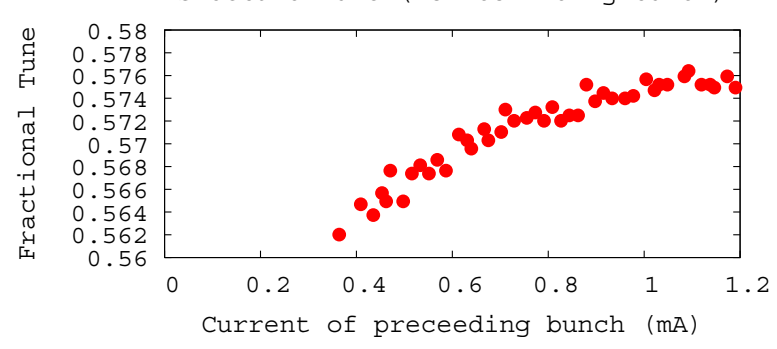

Figure 7: Sideband tune of non-colliding observer bunch for different currents of preceeding bunch

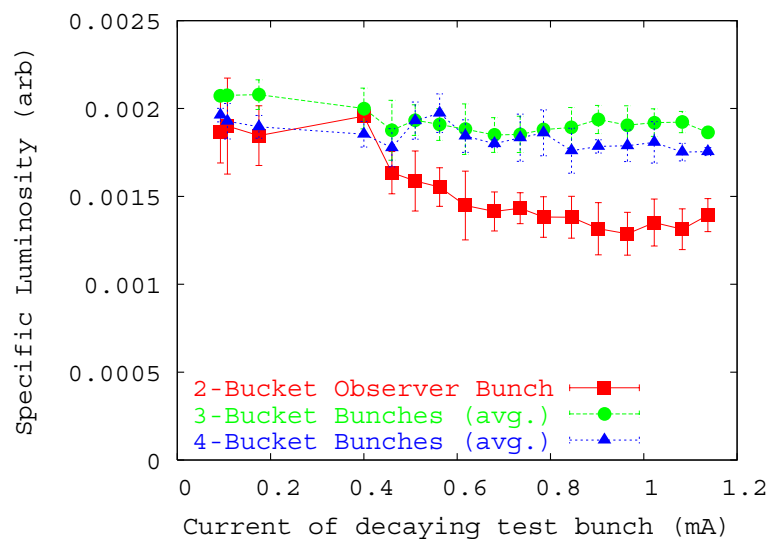

Figure 8: Specific luminosity of 2-bucket colliding observer bunch, and those of 3- and 4-bucket bunches, for different currents of the test bunch preceeding the 2-bucket observer bunch

than that of the 3- and 4-bucket bunches for test bunch currents above $\sim 0.4 \mathrm{~mA}$. This is again consistent with the sideband peak-height data.

\section{CONCLUSIONS}

Vertical betatron sideband peaks are found in closelyspaced bunches above certain current thresholds. The sidebands are smeared out when the bunches are in collision. The thresholds for sideband appearance match the thresholds for specific luminosity loss of the closely-spaced bunches relative to more widely-spaced bunches. This provides evidence that the luminosity loss is indeed due to electron-cloud induced instability in the more closelyspaced bunches, and indicates a cloud-induced limit to the practical packing density of bunches in the rings.

\section{REFERENCES}

[1] J.W. Flanagan, K. Ohmi, H. Fukuma, S. Hiramatsu, M. Tobiyama and E. Perevedentsev, Phys. Rev. Lett. 94, 054801 (2005).

[2] H. Ikeda et al., elsewhere in these proceedings. 